\title{
Analysis of Decoupling of Economic Growth and Resource Consumption in China's Marine Fishery
}

\author{
Zixiao Su1, Yannan Wang ${ }^{2 *}$, Jianwen $\mathbf{J i}^{2}$ \\ ${ }^{1}$ Information Engineering Department of Yantai Gold College, Yantai, China \\ ${ }^{2}$ Center for Studies of Marine Economy and Sustainable Development of Liaoning Normal University, Dalian, China \\ Email: ^yannan1916@163.com
}

How to cite this paper: Su, Z. X., Wang, Y. N., \& Ji, J. W. (2020). Analysis of Decoupling of Economic Growth and Resource Consumption in China's Marine Fishery. Journal of Geoscience and Environment Protection, 8, 10-21.

https://doi.org/10.4236/gep.2020.83002

Received: February 20, 2020

Accepted: March 7, 2020

Published: March 10, 2020

\begin{abstract}
The marine fishery resources are declining with the increase of fishing intensity, and the sustainable development of marine fishery economy has become the focus of attention. In this paper, the economic loss of marine natural fishery resources is taken as the undesired output. The SBM model is used to evaluate the economic efficiency of marine fisheries in 11 provinces and cities in China's coastal areas from 2004 to 2015. Based on this, decoupling theory is adopted to analyze the spatial and temporal evolution pattern of marine fishery economic efficiency and resource consumption in various provinces and cities. The results show that: 1) Based on the perspective of time and space, the economic efficiency of marine fisheries in 11 provinces and cities along the coast of China is polarized and has not been effectively improved, indicating that the level of fishery economic development in China's coastal areas is unbalanced; 2) Based on the overall perspective, the overall economic efficiency of China's marine fisheries is low, indicating that China's marine fishery economy is developing at a slower rate; 3) Based on the perspective of decoupling theory, the economic efficiency of marine fisheries in China and the consumption of fishery resources have shown a trend in the research period. The structure of China's marine fishery industry, which is dominated by traditional fisheries, needs to be adjusted.
\end{abstract}

\section{Keywords}

Economic Efficiency of Marine Fisheries, Undesired Output, SBM, Decoupling Theory

\section{Introduction}

Since the $21^{\text {st }}$ century, as the world has enters the ocean century, marine re- 
sources have received more and more attention. The exploitation and utilization of marine resources has become an increasingly important part of the marine strategies of all countries in the world, marine fisheries, as an important part of marine resources, are playing an increasingly important role. The over-exploitation of marine fishery resources and the exhaustion of fishery resources have become a common problem all over the world, especially for coastal countries and regions. Countries have proposed different solutions to the current situation of fishery resources and in combination with their own actual conditions: Canada mainly controls the catch of fishery resources through quota system and fishing boat subsidies; Japan uses the fishery rights system and the fishery license system to control the catch of fishery resources through local community autonomies; The United States limits the catch of fishery resources by setting up observers and the Total Allowable Catches (TAC) system. Through different means and measures, China has contributed "China's wisdom" in solving the dilemma of fishery resources: As of 2017, China's central and local governments have issued 287 laws and regulations on fisheries, and 1173 laws and regulations concerning fisheries (data from the legal and regulatory information base). Overfishing has been effectively controlled to a certain extent, but China's fishery economy is mostly concentrated in traditional industries, and the fishery economy is inefficient. Large-scale fishery resources development leads to serious pollution in the sea area, and undesired output from fishery economic production activities, it has hindered the development of China's fishery economy. The sustainable development of fishery resources is in urgent need of scientific analysis. Through the research on the literature, it is found that the fishery resources are significantly affected by the government's administrative intervention in the process of fishery economic development (Till, 2010). The government can help the development of fishery economy through financial policies and administrative laws to promote the improvement of domestic fishery economic efficiency. Among them, Kleih et al. (2013) analyzed the financial support of developing countries, and believed that the development of the financial system can improve the economic development of small and medium-sized fishermen's businesses. Brick et al. (1994) analyzed the risk attitudes of a large number of people in various fishing communities on the west coast of South Africa, and found that fishery policies could significantly influence the awareness of legal evasion of female fishermen. Zengin Fawzi et al. (2018) explained the importance of regional sea area cooperative management by analyzing the status of fishery fishing in the Black Sea region. The above-mentioned literature mostly analyzes the development of fishery economy through the perspective of management, and does not make a detailed analysis of the economic efficiency of fishery.

The analysis of the economic efficiency of fishery can effectively analyze the fishery economy through the technical and economic efficiency layer: Yang \& Zhou (2014) use DEA model to analyze the production efficiency of Chinese fishery science and technology, and demonstrate the high investment in the 
process of technological innovation in China. The problem is that the efficiency of fisheries for pure science and technology has not reached the effective level of scale. $\mathrm{Xu}$ (2015) studied the level and regional convergence of green growth of Marine fishery under the constraint of environment in China, and confirmed that China's Marine fishery economy is an extensive type of economy. The growth rate of green total factor productivity of dynamic Marine fishery in most provinces and cities is far lower than that of Marine fishery GDP. Gao \& Chen (2003) based on the scarcity theory of resources, confirmed the continuous reduction of offshore fishing grounds and Marine fishery resources and the decline of the economic efficiency of fishery production factors, which has become the main factor restricting the sustainable development of China's marine fisheries. Based on the traditional DEA model, the efficiency relationship between production factors and expected output (generally the total output of fishery economy) is mainly studied from the perspective of performance evaluation of input and output of Marine fishery economy or the development level of fishery economy. It is worth noting that the Marine environment pollution caused by fishery and its related industries is becoming increasingly serious, which seriously damages the economic benefit of fishery. Therefore, it is more objective and scientific to consider undesired output, such as pollution, when measuring the economic efficiency of fisheries. This paper constructs an SBM-DEA model that considers undesired outputs, and measures the economic efficiency of marine fisheries in 11 provinces and municipalities in China's coastal areas from 2004 to 2015, and describes the evolution of its spatial and temporal patterns. On the basis of analyzing the distribution characteristics of time and space patterns in various regions, using the decoupling theory, the economic efficiency and resource consumption of marine fisheries during the "Eleventh Five-Year Plan" (2007-2010) and "Twelfth Five-Year Plan" (2011-2014) The relationship is measured and analyzed for its evolution, in order to provide reference for the economic development of marine fisheries.

\section{Variable Selection, Data Sources and Model Methods}

\subsection{Variable Selection and Data Source}

The accuracy of measuring the efficiency results using the Data Envelopment Analysis (DEA) method depends to a large extent on the input and output indicators used in the evaluation process (Zhang et al., 2017). In terms of input indicators, according to western economics, land, labor and capital are usually the most basic factors of production (Gregory Mankiw, 1977). In terms of marine fishery economic input indicators, marine fishery economic development is highly dependent on resource endowments and marine environment, so the input of marine resources and environment is crucial for the development of marine fisheries. Drawing on the existing research results (Guan et al., 2013; Chen et al., 2010; Chen et al., 2014), considering that the traditional fishery industry still accounts for a large proportion of China's marine fishery industry structure, 
this paper will use the coastal provinces of 11 provinces (excluding Hong Kong, Macao and Taiwan) as decision-making units. The aquaculture area, the end of the fishing vessel and the marine fishery labor force are used as input variables. In terms of output indicators, the total output value of marine fisheries is selected as the expected output. In marine fishery activities, not only the expected output will be produced, but also undesired output such as wastewater and waste will be generated, causing certain losses to the output value of marine fishery. Based on the "China Fishery Eco-Environmental Bulletin" (Ministry of Agriculture, 2004-2015), the estimation principle of the economic loss of marine natural fishery resources, according to the estimation method of the economic loss of natural fishery in the bulletin, the specific calculation of the 11 provinces and municipalities in China's coastal areas from 2004 to 2015 Unexpected output value of natural fishery economy as a quantitative indicator of undesired output (in view of the unmeasurable nature of undesired output of marine aquaculture, this study does not include the economic loss of this part, so theoretically the economic efficiency of China's marine fisheries, the value will be lower than the results of this study). The data of input variables and expected output variables are from China fishery statistical yearbook (Fisheries Bureau of the Ministry of Agriculture, 2004-2015), and the output value of Marine fishery is calculated with 2000 as the base period (2014 National Fisheries Economic Statistics Bulletin, 2015). The quantitative evaluation index system of Marine fishery economic efficiency is shown in Table 1.

\subsection{Non-Expected Output of SBM Model}

Data Envelope Analysis (DEA) has proven to be a very effective tool in measuring the efficiency of decision-making units (Charnes, Cooper, \& Rhodes, 1978). The application of the traditional DEA method mainly focuses on radial or angle models such as CCR (Charnes, Cooper, \& Rhodes, 1978) and BBC (Banker, Charnes, \& Cooper, 1984). The output of these models is mostly based on desirable output, and not adequately consider the redundancy and relaxation of inputs and outputs, it also fails to accurately measure the efficiency values when there are undesirable output (Zhao et al., 2016).

Table 1 . The index system of marine fishery economic efficiency.

\begin{tabular}{llll}
\hline Target layer & Reference layer & & Indicator layer \\
\hline $\begin{array}{l}\text { Marine Fishery } \\
\text { Economic Efficiency }\end{array}$ & Input Indicators & Natural resource input & Mariculture area/(ha) \\
& & Capital Investment & Year-end ownership of fishing vessels/(t) \\
& Undesirable Outputs Indicators & Labor input & Marine fishery labor force/(person) \\
& Desirable Outputs Indicators & Negative effects of natural marine fishery & $\begin{array}{l}\text { Losses in Marine Natural Fishery } \\
\text { (Estimation of fishery water pollution)/(t) }\end{array}$ \\
& & & Marine Fishery Output/(million)
\end{tabular}

Note: We use mariculture area as alternative indicator of natural resource input is due to the fact that mariculture area is the best indicator that can be obtained in place of offshore sea area, and others are not specific to the interprovincial level. 
Tone proposed a SBM (Slacks-based measure) model based on relaxation measures to deal with undesirable output (Tone, 2003). We used the SBM model proposed by Tone that considers undesirable output can be expressed as:

$$
\begin{gathered}
p=\frac{1-\frac{1}{m} \sum_{i=1}^{m} \frac{s_{i}^{-}}{x_{i 0}}}{1+\frac{1}{s_{1}+s_{2}}\left(\sum_{r=1}^{s_{1}} \frac{s_{r}^{g}}{y_{r 0}^{g}}+\sum_{r=1}^{s_{2}} \frac{s_{r}^{b}}{y_{r 0}^{b}}\right)} \\
\text { s.t. }\left\{\begin{array}{l}
x_{0}=X \lambda+s^{-} \\
y_{0}^{g}=Y^{g} \lambda-s^{g} \\
y_{0}^{b}=Y^{b} \lambda-s^{b} \\
s^{-} \geq 0, s^{g} \geq 0, s^{b} \geq 0, \lambda \geq 0
\end{array}\right.
\end{gathered}
$$

The variable in the formula(1) can be explained as follows: $s=\left(s^{-}, s^{g}, s^{b}\right)$ represent slack variables for input, desirable output, and undesirable output respectively; $\lambda$ was the weight vector; $p$ was the objective function, the variable $s^{-}, s^{g}, s^{b}$ were strictly decreasing and the range of it was $0 \leq \rho^{*} \leq 1$. For each evaluated decision-making unit, if and only if $\rho^{*}=1$ which was $s^{-}=0, s^{g}=0, s^{b}=0$, the evaluated decision-making unit is efficient; if so $\rho^{*}<1$, it means that the evaluated decision-making unit is inefficient and there is still have improvement in input and output (Tone, 2003). For ease to analysis, refer to the study of Ma Zhanxin's. Set the efficiency value $\rho^{*}=1$ as the highest efficiency; $0.8 \leq \rho^{*}<1$ as the efficiency is good; $0.6 \leq \rho^{*}<0.8$ as the efficiency is medium; $\rho^{*}<0.6$ as the efficiency is invalid.

\subsection{Decoupling Theory and Its Model}

Decoupling is widely used in different fields. The Organization for Economic Co-operation and Development (OECD) initially applied the concept of "decoupling" to the field of agricultural policy and analyzed the interrelationship between policy and trade and market equilibrium. The World Bank then introduced the concept into the resource and environment to analyze resource consumption and the decoupling between environmental pollution and economic growth.

There are three main methods of decoupling measurement in the academic world: one is the decoupling factor proposed by OECD; Secondly, the decoupling elastic coefficient proposed by Tapio; Thirdly, the decoupling evaluation method based on IPAT equation. Due to Tapio decoupling elastic model is more suitable for analysis of resources and the relationship between the economic development, this paper adopts the model decoupling fishery resources degradation and economic growth model was constructed and fishery resources use efficiency and economic growth "hook" model, discusses the 11 provinces, coastal fisheries resources and economic growth exist concrete development trend of space and time differentiation (Kleih et al., 2013).

The specific model is as follows: 
The GDP elasticity of fishery resource consumption $=$ the percentage change in fishery consumption/the percentage change in

$$
G D P=E_{C C}=\% \triangle C C / \% \Delta G D P
$$

GDP elasticity of coal utilization efficiency $=$ percentage of change in fishery resource utilization efficiency/percentage of change in

$$
G D P=E_{C E}=\% \triangle C E / \% \triangle G D P
$$

The judgment principle is:

$E_{C C}, E_{C E}>1$ for absolute linkage, $E_{C C}, E_{C E}=1$ is the critical point of absolute linkage and relative decoupling, $0<E_{C C}, E_{C E}<1$ is relative decoupling, $E_{C C}, E_{C E}<0$ is absolute decoupling.

\section{Analysis of Spatial Temporal Differences of Economic Efficiency}

Based on the above model, we used MaxDEA 6.4 software to estimate the economic efficiency of marine fishery considering the undesirable output (SBM) in the 11 coastal provincial-level areas from 2004 to 2015 (Table 2). The results showed that the overall economic efficiency of marine fishery was at a relatively low level during this period.

Based on the SBM model considering undesirable output, based on the economic efficiency of marine fishery in 11 coastal provincial-level areas in China from 2004 to 2015, cross-sectional data for 2004, 2009, and 2015 were selected, and ARCGIS 10.0 was used for spatial-temporal analysis of the economic efficiency of marine fishery and mapping of the distribution, see Figure 1.

1) In general, the number and area of provinces and cities ranked first in terms of efficiency values have remained basically unchanged; the number and

\begin{tabular}{|c|c|c|c|c|c|c|c|c|c|c|c|c|}
\hline Area & 2004 & 2005 & 2006 & 2007 & 2008 & 2009 & 2010 & 2011 & 2012 & 2013 & 2014 & 2015 \\
\hline Tianjin & 1.000 & 1.000 & 1.000 & 1.000 & 1.000 & 1.000 & 1.000 & 1.000 & 1.000 & 1.000 & 1.000 & 1.000 \\
\hline Hebei & 0.222 & 0.269 & 0.244 & 0.169 & 0.140 & 0.121 & 0.159 & 0.167 & 0.235 & 0.195 & 0.248 & 0.238 \\
\hline Liaoning & 0.362 & 0.421 & 0.429 & 0.403 & 0.314 & 0.289 & 0.298 & 0.259 & 0.264 & 0.205 & 0.236 & 0.277 \\
\hline Shanghai & 1.000 & 1.000 & 1.000 & 1.000 & 1.000 & 1.000 & 1.000 & 1.000 & 1.000 & 1.000 & 1.000 & 1.000 \\
\hline Jiangsu & 1.000 & 1.000 & 1.000 & 1.000 & 1.000 & 1.000 & 1.000 & 1.000 & 1.000 & 1.000 & 1.000 & 1.000 \\
\hline Zhejiang & 0.706 & 0.930 & 0.972 & 0.983 & 0.981 & 0.956 & 0.922 & 0.940 & 0.926 & 0.873 & 0.950 & 0.964 \\
\hline Fujian & 1.000 & 1.000 & 1.000 & 0.991 & 1.000 & 0.989 & 0.995 & 0.994 & 0.996 & 0.990 & 0.984 & 0.988 \\
\hline Shandong & 0.729 & 0.966 & 1.000 & 0.888 & 0.781 & 0.849 & 1.000 & 0.869 & 1.000 & 1.000 & 1.000 & 1.000 \\
\hline Guangdong & 0.407 & 0.795 & 0.928 & 0.928 & 0.764 & 0.924 & 0.889 & 0.803 & 0.836 & 0.548 & 0.728 & 0.812 \\
\hline Guangxi & 0.311 & 0.534 & 0.282 & 0.257 & 0.281 & 0.318 & 0.563 & 0.299 & 0.383 & 0.314 & 0.262 & 0.387 \\
\hline Hainan & 1.000 & 1.000 & 1.000 & 1.000 & 1.000 & 1.000 & 1.000 & 1.000 & 1.000 & 1.000 & 1.000 & 1.000 \\
\hline The national & 0.620 & 0.752 & 0.719 & 0.678 & 0.639 & 0.646 & 0.706 & 0.648 & 0.695 & 0.628 & 0.661 & 0.700 \\
\hline
\end{tabular}

Table 2. The marine fishery economic efficiency value based on SBM model with undesirable output from 2004 to 2015. 


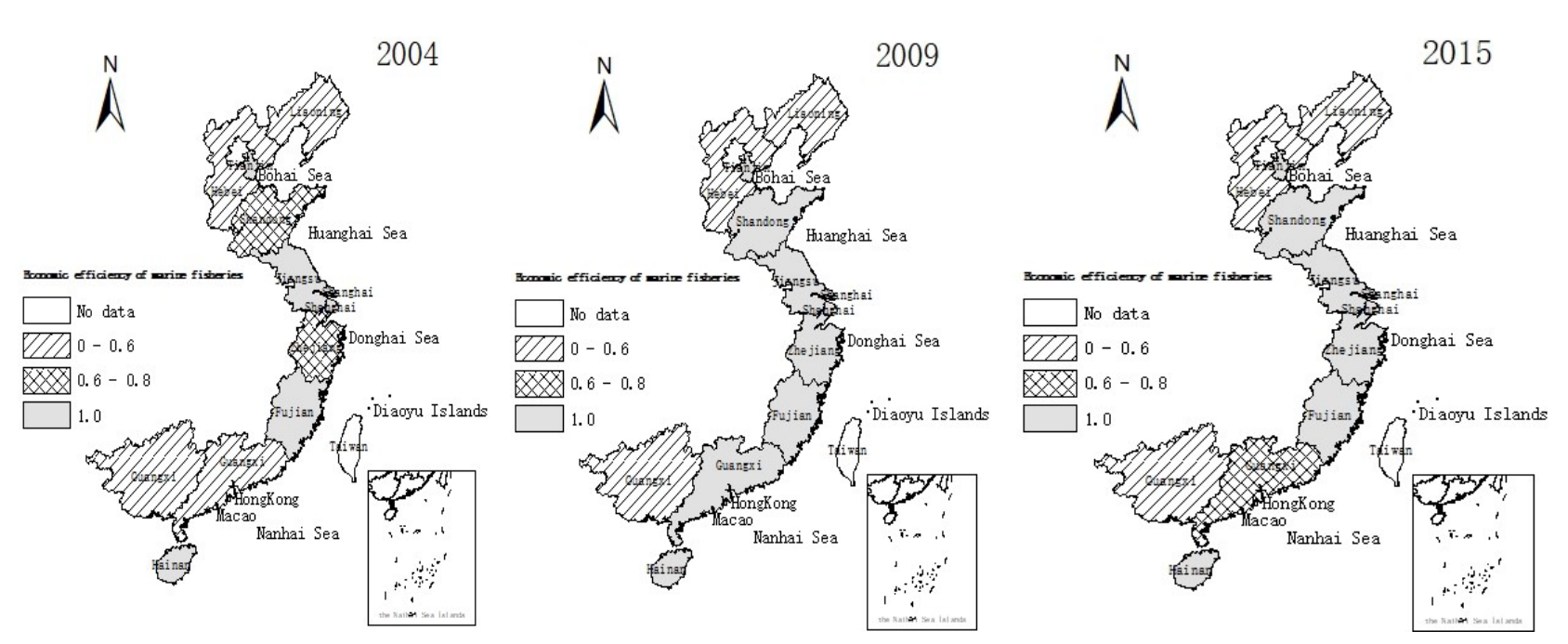

Figure 1. The spatial evolution of marine fishery economic efficiency with considering undesirable outputs.

area of provinces and cities ranked the fourth in terms of efficiency values have remained basically unchanged; Fluctuations. From the spatial evolution of the three sample intervals, the polarization has not improved.

2) Spatial pattern of economic efficiency of marine fisheries. According to the characteristics of the economic efficiency of marine fisheries in various provinces and municipalities over the years, the 11 coastal provinces and urban areas are divided into four types. a) Efficient and stable type. Tianjin, Shanghai, Jiangsu and Hainan are. b) Medium efficiency stable type. Zhejiang and Fujian are. c) Inefficient and stable. Hebei, Liaoning and Guangxi are. d) Medium efficiency fluctuations type. Shandong and Guangdong are.

Based on the comprehensive time series analysis and time-space difference analysis, the geometrical average economic efficiency of China's marine fisheries is maintained at around 0.6, indicating that the overall economic efficiency of China's marine fisheries is low; the high-efficiency areas are rising from the beginning of the six to the end of the seven, low The effect area was reduced from 5 at the beginning of the period to 4 at the end of the period. Overall, the polarization situation of marine fisheries economic development in 11 provinces and cities in China's coastal areas has not been significantly improved.

\section{Decoupling Analysis of Resources, Utilization Efficiency and Economic Growth}

Combined with the decoupling model and related discriminant division for 2007 (the yearbook statistics in 2007 began to change, the previous data cannot be unified calculation, so in the decoupling analysis, we select 2007 as the starting year) - 2014 China's fishery economic growth and resources the decoupling relationship between consumption and utilization efficiency is decoupled from time series and spatial evolution. In terms of time, with reference to the five-year planning time schedule for national economic and social development, based on available data, take the 2007-2010 ( $11^{\text {th }}$ Five-Year Plan, due to data limited de- 
fault 2006) and 2011-2014 (According to the efficiency value of SBM and the decoupling model, the decoupling relationship between fishery resources, utilization efficiency and economic growth is calculated as shown in Table 3.

\subsection{Decoupling Time Series Analysis}

According to the results of the decoupling calculation, during the "Eleventh Five-Year Plan" period,

1) The economic growth of China's marine fisheries and the consumption of fishery resources are absolutely decoupled, that is, the growth of the fishery economy is based on the consumption of lower fishery resources. It shows that China's fishery industry structure is relatively reasonable during the "Eleventh Five-Year Plan" period, and the contribution rate of fisheries secondary and tertiary industries to fishery economic growth is relatively high.

2) The analysis results also show that the utilization efficiency of fishery resources is subject to the large base of fishing vessels and the large total labor force of the fishery, showing an absolute hook. The economic efficiency of fisheries directly affects the state of fishery economic growth. The economic efficiency of marine fisheries fluctuated greatly in 2008-2009, which was 0.631 and 0.600 respectively. In 2010, it rebounded to 0.661 . The total economic output

Table 3. Analysis on the decoupling of marine fishery resources, fisheries economic efficiency and economic growth during 2007-2014.

\begin{tabular}{|c|c|c|c|c|c|c|c|}
\hline $\begin{array}{l}\text { Research interval, } \\
2007-2010\end{array}$ & $\mathrm{E}_{\mathrm{CC}}$ & $\mathrm{E}_{\mathrm{CE}}$ & Decoupling state & $\begin{array}{l}\text { Research interval, } \\
2011-2014\end{array}$ & $\mathrm{E}_{\mathrm{CC}}$ & $\mathrm{E}_{\mathrm{CE}}$ & Decoupling state \\
\hline Tianjin & 0.595 & 0.000 & $\begin{array}{l}\text { Relative decoupling/ } \\
\text { Absolute decoupling }\end{array}$ & Tianjin & -3.730 & 0.000 & $\begin{array}{l}\text { Relative decoupling/ } \\
\text { Absolute decoupling }\end{array}$ \\
\hline Hebei & -0.012 & -124.614 & Absolute decoupling & Hebei & -0.784 & 3.243 & $\begin{array}{l}\text { Absolute decoupling/ } \\
\text { Absolute hook }\end{array}$ \\
\hline Liaoning & -0.223 & 0.64 & $\begin{array}{l}\text { Absolute decoupling/ } \\
\text { Absolute hook }\end{array}$ & Liaoning & -0.048 & -1.771 & Absolute decoupling \\
\hline Shanghai & -1.463 & 0.000 & Absolute decoupling & Shanghai & -0.219 & 0.000 & Absolute decoupling \\
\hline Jiangsu & -0.016 & 0.000 & Absolute decoupling & Jiangsu & -0.020 & 0.000 & Absolute decoupling \\
\hline Zhejiang & -1.150 & -0.070 & Absolute decoupling & Zhejiang & 0.451 & 0.114 & Relative decoupling \\
\hline Fujian & -0.212 & -1.624 & Absolute decoupling & Fujian & 0.169 & -3.125 & $\begin{array}{l}\text { Relative decoupling/ } \\
\text { Absolute decoupling }\end{array}$ \\
\hline Shandong & -0.045 & -20.755 & Absolute decoupling & Shandong & -0.142 & -14.139 & Absolute decoupling \\
\hline Guangdong & -0.122 & -0.599 & Absolute decoupling & Guangdong & 0.101 & 17.090 & $\begin{array}{l}\text { Relative decoupling/ } \\
\text { Absolute hook }\end{array}$ \\
\hline Guangxi & -1.402 & -116.533 & Absolute decoupling & Guangxi & -0.041 & 37.966 & $\begin{array}{l}\text { Absolute decoupling/ } \\
\text { Absolute hook }\end{array}$ \\
\hline Hainan & 0.094 & 0.000 & $\begin{array}{l}\text { Relative decoupling/ } \\
\text { Absolute decoupling }\end{array}$ & Hainan & 0.729 & 0.000 & $\begin{array}{l}\text { Relative decoupling/ } \\
\text { Absolute decoupling }\end{array}$ \\
\hline Mean & -0.057 & -0.132 & $\begin{array}{l}\text { Absolute decoupling/ } \\
\text { Absolute hook }\end{array}$ & Mean & 0.189 & 5.068 & $\begin{array}{l}\text { Relative decoupling/ } \\
\text { Absolute hook }\end{array}$ \\
\hline
\end{tabular}


increased from 95.391 billion yuan in 2007 to 1292.471 billion yuan in 2010, resulting in fishery utilization efficiency and the absolute state of economic growth.

3) The consumption of fishery resources and economic growth have fallen back to a relative decoupling state. The consumption dependence of fishery resources has increased compared with the "Eleventh Five-Year Plan" period. Due to the continuous decline of natural fishery resources, the catch of fishery farming has been increasing, resulting in the total amount of fishery catches. Substantial growth. The " $13^{\text {th }}$ Five-Year Plan" will control domestic marine catches to within 10 million tons, while in 2016 China's marine fishery catches will be 1328.27 tons. It is necessary to control the catch, control the number of fishing vessels, and reduce fishermen to achieve the " $13^{\text {th }}$ Five-Year" fishery. Planning objectives.

4) The utilization efficiency of fishery resources and economic growth are still absolutely linked. Although the total amount of fishing continues to increase, the cumulative effect of the ship-to-production policy during the "Eleventh Five-Year Plan" period, the base of fishing vessels during the "Twelfth Five-Year Plan" period. With the reduction of the total labor force of the fishery, the "one increase and two reductions" keep the state of fishery efficiency and economic growth absolutely linked.

\subsection{Decoupling Spatial Sequence Analysis}

Combined with the decoupling degree criterion and the decoupling results of fishery economic growth and fishery efficiency in the coastal provinces of the eleventh province, the analysis is as follows:

1) During the "Eleventh Five-Year Plan" period, the fishery consumption and economic growth in China's coastal areas are relatively decoupled: Tianjin and Hainan provinces and cities, the state distribution is scattered. Hainan is at the edge of relative decoupling, close to absolute decoupling; Tianjin has used its economic progress to complete the absolute decoupling process of fishery consumption and economic growth. After the implementation of the policy of shifting production to the industry during the "Eleventh Five-Year Plan" period, the number of areas where fishery consumption and economic growth showed relative decoupling during the "Twelfth Five-Year Plan" period increased to four, namely Zhejiang, Fujian, Guangdong and Hainan. Although Hainan is still in a relatively decoupling state, it has a tendency to turn into an absolute link. It shows that the growth of the fishery economy has begun to deviate from the dependence on resources, and the effect of promoting the sustainable use of resources, such as the conversion of production and transformation, has been highlighted. Due to the implementation of the relevant policies of the government to change production and environmental protection, some fishermen have successfully switched production to work, and the efficiency of labor has increased, resulting in an absolute decoupling of fishery resources and economic growth 
(Till, 2010).

2) During the "Eleventh Five-Year Plan" period, the provinces and cities with absolute decoupling of fishery consumption and economic growth: Hebei, Liaoning, Shanghai, Jiangsu, Zhejiang, Fujian, Shandong, Guangdong, and Guangxi. Tianjin has changed from the relative decoupling during the $11^{\text {th }}$ Five-Year Plan period to the absolute decoupling during the $12^{\text {th }}$ Five-Year Plan period. The rapid development of the fishery secondary industry has played a major role in promoting trade (export, port construction). Zhejiang, Fujian, and Guangdong changed from the absolute decoupling during the $11^{\text {th }}$ Five-Year Plan period to the relative decoupling during the $12^{\text {th }}$ Five-Year Plan period, and the total amount of marine aquaculture increased rapidly.

3) During the "Eleventh Five-Year Plan" period, It was Liaoning Province that with absolute decoupling of fishery utilization efficiency and economic growth. This means that fishery utilization efficiency in Liaoning grew faster than the economic growth. Tianjin, Shanghai, Jiangsu, Zhejiang, Fujian, Shandong, Guangxi, Guangdong, and Hainan showed absolute decoupling status. This was because Tianjin, Zhejiang, Fujian, and Shandong experienced negative growth in fishery utilization efficiency during this period, with zero growth in Shanghai, Jiangsu, and Hainan. During the "Twelfth Five-Year Plan" period, Hebei, Guangdong, and Guangxi were absolutely linked: Guangdong's fishery utilization efficiency and economic growth showed a state of absolute decoupling and an absolute link, indicating that Guangdong's fishery economic growth mode has retreated during this period. Phenomenon; Guangxi changed from the absolute decoupling to the absolute hook state; Liaoning, Shanghai, Jiangsu, Zhejiang, Fujian, and Shandong showed absolute decoupling; Tianjin and Hainan changed from absolute decoupling to relative decoupling. The overall trend indicates that the sustainable development trend of fisheries during the "Eleventh Five-Year Plan" period is better than that during the "Twelfth Five-Year Plan" period. During the "Twelfth Five-Year Plan" period, China's overall fishery economy is developing at a faster rate, but the fishery industry is slow to adjust, and the primary industry accounts for. This has led to a reversal of the overall sustainable development trend. In the overall development, the sustainable development level of marine fisheries in the "Twelfth Five-Year Plan" needs to be improved, which is consistent with the " $13^{\text {th }}$ Five-Year Plan for National Fisheries Development".

\section{Conclusion}

In this paper, the SBM model which considers undesired output is used to measure the economic efficiency of marine fisheries in the coastal provinces of 11 provinces from 2004 to 2015, and comparative analysis is carried out. Considering that it's having certain objectivity the economic efficiency value of marine fishery undesired output reflects the change of economic efficiency of marine fishery under the condition of marine environmental pollution. Therefore, this 
paper selects the economic loss of marine natural fishery resources caused by water pollution as the index of undesired output. The GIS space technology is used to describe the temporal and spatial patterns' changes of historical efficiency values of coastal provinces and cities in the condition of considering undesired output. On this basis, using the decoupling theory to measure the sustainable development level of the fishery economy, the following conclusions are drawn:

1) Analysis of time and space differences, the overall efficiency of China's fishery economy development is low, reflecting the unsatisfactory effect of China's relevant marine fishery economic regulation in recent years. From the perspective of 11 provinces and municipalities, China's marine fishery economic efficiency is significantly different between regions, showing an imbalanced feature, indicating that the polarization of fishery economic development in 11 provinces and cities in China has not been effectively improved.

2) From the theoretical analysis of decoupling, overall, sustainable development of China's marine fishery has shown a downward trend during the "Eleventh Five-Year Plan" and "Twelfth Five-Year Plan". The overall economic efficiency of fishery is more closely related to the consumption of fishery resources. Sustained development of marine fishery faces serious challenges during the research period, and the upgrading of the fishery industry structure is increasingly important. In the "Thirteenth Five-Year Plan", China's marine fishery with traditional fishery as the main mode of production needs to further adjust the proportion of the primary industry.

\section{Acknowledgements}

We would like to acknowledge the financial support from the Social Science Fund Project of Liaoning province (L19BJ006) and the Humanities and social science research project of education department of Liaoning province (WJ2019016).

\section{Conflicts of Interest}

The authors declare no conflicts of interest regarding the publication of this paper.

\section{References}

(2015). 2014 National Fisheries Economic Statistics Bulletin. China Fisheries, No. 6, 14-15.

Banker, R. D., Charnes, A., \& Cooper, W. W. (1984). Some Models for Estimating Technical and Scale Inefficiencies in Date Envelopment Analysis. Management Science, 30, 1078-1092.

Brick, K., Yisser, M., \& Burns, J. (1994). Risk Aversion: Experimental Evidence from South African Fishing Communities. American Journal of Agricultural Economics, 94, 133-152. https://doi.org/10.1093/ajae/aar120

Charnes, A., Cooper, W. W., \& Rhodes, E. (1978). Measuring the Efficiency of Decision Making Units. European Journal of Operational Research, 2, 429-444. 


\section{https://doi.org/10.1016/0377-2217(78)90138-8}

Chen, X.-J., Liu, J.-L., Guan, W.-J. et al. (2014). Research and Application of Bio-Economic Model of Fishery Resources. Journal of Shanghai Ocean University, 23, 608-617.

Chen, Z. Z., Lin, Z. J., \& Qiu, Y. S. (2010). Evaluation of Sustainable Utilization of Fishery Resources in the South China Sea Based on AHP. Journal of Natural Resources, 25, 249-257.

Fisheries Bureau of the Ministry of Agriculture. (2004-2015). China Fisheries Statistical Yearbook. Ocean Publishing House.

Gao, J., \& Chen, D. Q. (2003). Effects of Fisheries Production Factors on the Sustainable Development of Chinese Marine Fisher Economics. Journal of Shanghai Fisheries University, 12, 86-92.

Gregory Mankiw, N. (1977). Principle of Economics. London: Oxford University Press.

Guan, W. J., Tian, S. Q., Zhu, J. F. et al. (2013) Research Status and Prospects of Fishery Resource Assessment Model. Chinese Journal of Fisheries Sciences, No. 5, 1112-1120. https://doi.org/10.3724/SP.J.1118.2013.01112

Kleih, U., Linton, J., Marr, A. et al. (2013). Financial Services for Small and Medium-Scale Aquaculture and Fisheries Producers. Marine Policy, 37, 106-114. https://doi.org/10.1016/j.marpol.2012.04.006

Ministry of Agriculture (2004-2015). Ministry of Environmental Protection. China's Fisheries Eco-Environment Announcement.

Till, M. (2010). Towards Sustainable Fisheries Subsidies: Entering a New Round of Reform under the Common Fisheries Policy. Marine Policy, 34, 1117-1124.

https://doi.org/10.1016/j.marpol.2010.03.011

Tone, K. (2003). Dealing with Undesirable Outputs in DEA: A Slacksbased Measure (SBM) Approach. GRIPS Research Report Series.

$\mathrm{Xu}, \mathrm{W}$. (2015). Research on the Change of Green Total Factor Productivity and Regional Convergence of Marine Fisheries in China. Ocean University of China.

Yang, W., \& Zhou, W. (2014). An Empirical Analysis of Fisheries Science and Technology Production Efficiency Based on DEA Model. Chinese Agricultural Science Bulletin, 30, 139-142.

Zengin Fawzi, M., Mihneva, V., \& Duzgunes, E. (2018). Analysing the Need of Communication to Improve Black Sea Fisheries Management Policies in the Riparian Countries. Turkish Journal of Fisheries and Aquatic Sciences, No. 1, 199-209.

Zhang, L. X., Zhu, D. L., Du, T. et al. (2017). Temporal and Spatial Pattern Evolution and Driving Factors of Urban Construction Land Use Efficiency Based on DEA Model. Resources Science, 39, 418-429.

Zhao, L., Zhang, Y. S., Jiao, X. X. et al. (2016). Study on China Ocean Economic Efficiency Evaluation Based on SBM and Malmquist Productivity Index. Resources Science, 38, 461-475. 